\title{
La elección de estudio superiores universitarios en estudiantes de último curso de bachillerato y ciclos formativos
}

\author{
Choosing Higher Education for Senior High School and Upper Education Students
}

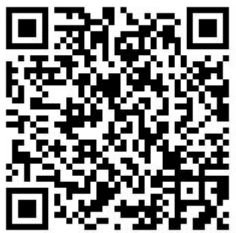

\author{
Asunción Martínez-Martínez ${ }^{1}$ \\ Universidad de Granada \\ Granada, España \\ asuncionmm@ugr.es \\ Félix Zurita-Ortega \\ Universidad de Granada \\ Granada, España \\ felixzo@ugr.es \\ Manuel Castro-Sánchez ${ }^{3}$ \\ Universidad de Granada \\ España \\ manue87@correo.ugr.es \\ Ramón Chacón-Cuberos 4 \\ Universidad de Granada \\ Granada, España \\ ramonchachoncuberos@correo.ugr.es \\ María Angustias Hinojo-Lucena ${ }^{5}$ \\ Universidad de Granada \\ Granada, España \\ marianhl@ugr.es \\ Tamara Espejo-Garcés ${ }^{6}$ \\ Universidad de Granada \\ Granada, España \\ tamaraeg@correo.ugr.es
}

\footnotetext{
${ }^{1}$ Profesora Doctora del Departamento de MIDE de la Universidad de Granada (España).

2 Profesor Doctor del Área de Corporal de la Universidad de Granada (España).

${ }^{3}$ Investigador del grupo HUM-238 de la Universidad de Granada (España).

${ }^{4}$ Investigador del grupo HUM-238 de la Universidad de Granada (España).

${ }^{5}$ Profesora del Departamento de Didáctica de la Organización Escolar de la Universidad de Granada (España).

${ }^{6}$ Investigador del grupo HUM-238 de la Universidad de Granada (España).
} 
doi: http://dx.doi.org/10.15359/ree.20-1.14

URL: http://www.una.ac.cr/educare

CORREO: educare@una.cr

Recibido 10 de marzo de 2015 • Corregido 11 de octubre de 2015 • Aceptado 17 de noviembre de 2015

Resumen. La elección de titulación universitaria para el próximo curso es un tema de interés para los estamentos educativos de educación superior, pues permiten dictaminar qué tipo de estudiantes acceden a los diversos grados universitarios; asimismo entre los factores que inciden de manera concisa están las calificaciones o el perfil personal, por tanto, planteamos el siguiente estudio con los objetivos de determinar las titulaciones más demandadas tanto con limitación, como sin ella, de alumnado de último curso de bachillerato y ciclos formativos de grado superior de Granada, así como describir las características académicas y sociodemográficas, y evaluar la influencia que tienen las calificaciones académicas, rama de estudios y género en la elección de grado universitario. Participaron un total de 1.164 estudiantes de último curso de bachillerato y ciclos formativos, lo cual permitió el registro y evaluación de las variables antes mencionadas. Los resultados mostraron que las titulaciones de medicina, magisterio e ingenierías son las más demandadas sin limitación de ningún tipo, mientras que con limitación cambia medicina por enfermería, asimismo estudiantes de último curso de Granada tenían una nota media de notable, los varones se decantan más por los ciclos formativos y presentan peores calificaciones y las mujeres por el bachillerato, con notas medias ligeramente superiores, quedando constatada la influencia que tienen las calificaciones académicas, rama de estudios y el género en la elección de titulación con limitación o sin esta, así ellas se postulan por su carácter más altruista en grados de ciencias de la salud y sociales con notas más altas, mientras que los estudiantes masculinos prefieren titulaciones más técnicas con peores calificaciones.

Palabras claves. Elección de carrera, bachillerato, ciclos formativos, estudiantes, calificación, grados.

Abstract. Choosing a university degree for next year is a topic of interest for educational levels of higher education, since they allow to decide what kind of students access the different university degrees; also among the factors that affect concisely are qualifications or personal profile, therefore we propose the following study with the objective to determine the most popular degrees with limitation, as without it, of senior high school and upper education students of Granada, as well as describe the academic and socio-demographic characteristics, and evaluate the influence academic qualifications, branch of studies and gender have in choosing college degree. A total of 1,164 students in last year of high school and upper education students participated, which allowed the recording and evaluation of the variables mentioned above. Results showed that medicine, teaching and engineering degrees are the most in demand without any kind of limitation, while with limitation it changes medicine for nursing, also senior students from Granada had a remarkable average note, males opt more for upper education training and show worse grades and women choose bachelor with slightly higher average grades, verifying the influence academic qualifications, branch of studies and gender have in choosing a degree with or without limitation. Thus, due to its more altruistic nature, female students postulate in health science and social degrees, with higher grades, while male students prefer more technical qualifications with worse grades.

Keywords. Choosing a career, bachelor's degree, upper education training, students, qualifications, grades. 
En la sociedad actual existe cada vez una mayor preocupación hacia los sujetos jóvenes en cuanto a su futuro se refiere. De esta forma, en décadas anteriores, llegar a los 18 años ocasionaba una doble perspectiva: por un lado, se situaban aquellos grupos de jóvenes que pretendían seguir su periodo formativo; mientras que el resto se sumergía en el contexto laboral. Actualmente, esta situación es una quimera, pues el lugar donde se contextualiza este trabajo sufre una importante carencia de empleo (Blanco, Mercado y Prado, 2012; Martínez y Zurita, 2014; Ruesga, Martín y Pérez, 2010; Viñuela-Jiménez, Rubiera-Morollón y Cueto, 2010), motivada por la crisis que está afectando a la población mundial, por lo que, ante la escasez de empleo, la juventud decide seguir formándose con estudios de tipo universitario.

En este sentido y siguiendo la afirmación plasmada por Martínez y Zurita (2014), deberíamos indicar que la orientación educativa es un elemento clave e indispensable dentro del contexto de la educación, puesto que es donde los grupos de estudiantes deberían aprender y adquirir los conocimientos de qué hacer y realizar en etapas posteriores, ya sea a corto o medio plazo, si bien es cierto que no siempre estas poblaciones reciben las nociones adecuadas, pues existe una descoordinación entre los centros de enseñanzas medias y los de enseñanza superior (Cepero, 2009; Domínguez, Álvarez y López, 2013; Llosa, Acin, Cragnolino y Lorenzatti, 2012). Lo que viene a decirnos, que la orientación no es solamente una guía conductora sino que sirve para que la persona se conozca así misma e identifique el mundo que la rodea, actuando en calidad ciudadana responsable, motivada y productiva no solo en una sociedad laboral sino también en su contexto personal, como señalan Mantero (2010) y Martínez y Zurita (2014).

Las diversas opciones para elegir una titulación, rama de conocimiento o inserción al mercado laboral vienen propiciadas por la toma de decisión que tenga la persona, de esta manera, Bisquerra (1992, p. 16, basándose en Spokane y Oliver, 1983), mencionan: "No puede haber ningún tema ... más bello que la cuestión de qué le gustaría ser a un hombre y en qué ocupación debería comprometerse y hasta dónde debería perseguir este objetivo tanto en la juventud como en la madurez", destacando un elemento esencial en esa definición que es la vocación, esta se encuentra postulada en numerosas teorías como revisa Martínez-Martínez (2013) en su trabajo de investigación.

Cuando se pretende ver la influencia que van a tener los intereses en la elección vocacional hay que ver dicha diferencia. Acuña Hernández (2004) que los intereses expresados son aquellos que hacen referencia a cuestiones ocupacionales específicas del tipo: ¿en qué profesión concreta te gustaría trabajar?, ¿qué carrera universitaria te gustaría estudiar cuando acabes el bachillerato? Martín-Moreno (1996) diferencia entre intereses intrínsecos (vivencial, vocacional y profesional) y extrínsecos (circunstanciales y fácilmente modificables), por tanto, se aprecia que la temática es bastante importante. 
doi: http://dx.doi.org/10.15359/ree.20-1.14

URL: http://www.una.ac.cr/educare

CORREO: educare@una.cr

Son numerosos los estudios que han descrito las opciones universitarias, pero casi siempre desde el ámbito del bachillerato (Cortés y Conchado, 2012; Santana, Feliciano y Jiménez, 2012), otros autores como Pérez, Cupani y Beltramino (2004) incluyen en sus estudios intereses y habilidades de una persona, por un lado, y los factores inherentes a su ambiente, por el otro. También Vence, Palomares y Sánchez (2000, en Cepero, 2009) y Villanueva y Sorribes (1995) citan parámetros de tipo social, biológico o económico, partiendo de los señalado en el RD 1892/20087, que regula la forma de acceso a estudios superiores universitarios y que plantea tres vías, aunque casi todos los estudios, como se ha señalado con anterioridad, se fundamentan en el acceso desde bachillerato.

Estudios como los de Cepero (2009) sobre el contexto vocacional, Lorente, et al. (2011) que indican la importancia de la posterior salida profesional tras la realización de la titulación o los de Gallo, González y Salinero (2010) y Cordón, Cordón y García (2012) que exponen sobre la motivación de elección o el estudio de Lorenzo-Moledo, Argos, Hernández y Vera (2014) que citan las formas de acceso a la universidad teniendo en cuenta las diversas opciones, constituyen trabajos encaminados a dar respuesta a las posibilidades de éxito y limitaciones, planteando un elemento esencial que es la conexión entre los estudios secundarios postobligatorios y los universitarios.

En el presente estudio se consideró importante verificar la influencia de las calificaciones y el género en la elección de los estudios universitarios con limitación o sin esta del próximo curso, dada la escasez de estudios que aglutinaban de forma conjunta a estudiantes de bachillerato y ciclos formativos ${ }^{8}$, la finalidad era constatar si la nota media y el género establecían patrones de posible acceso a estudios superiores. El presente estudio nos aporta cifras sobre lo que acontece en sujetos de 17 a 30 años en cuanto a la decisión académica de futuro inmediato. Los objetivos planteados son:

- Determinar las titulaciones más demandadas tanto con cómo sin limitación de alumnos de último curso de Granada así como describir las características académicas y sociodemográficas.

- Evaluar la influencia que tienen las calificaciones académicas, rama de estudios y de género en la elección de titulación con limitación o sin esta.

\footnotetext{
${ }^{7}$ Real Decreto 1892 (2008), por el que se regulan las condiciones para el acceso a las enseñanzas universitarias oficiales.

${ }^{8}$ Ciclos formativos, según lo establecido en el Real Decreto 676 (1993), es donde se fijaron los aspectos básicos del currículo que constituyen las enseñanzas mínimas de la formación profesional específica, tanto de grado medio como de grado superior. A partir de su entrada en vigor se empezaron a impartir los ciclos formativos, de ambos grados, organizándose la formación profesional específica en familias profesionales. Este cambio en la enseñanza exige una mayor disponibilidad de medios técnicos y va dirigida a estudiantes que en poco tiempo (entre cuatro y seis trimestres) han de adquirir los conocimientos y habilidades necesarias para su incorporación al mundo laboral.
}

4

Asunción Martínez-Martínez, Félix Zurita-Ortega, Manuel Castro-Sánchez, Ramón Chacón-Cuberos, María Angustias Hinojo-Lucena y Tamara Espejo-Garcés (c) (1) (3) 


\section{Método}

El estudio presentó un diseño metodológico de carácter exploratorio, cuantitativo, descriptivo y de tipo transversal.

\section{Muestra}

En este trabajo de investigación se parte de una población general de 6.132 estudiantes que se encontraban cursando el último curso de las enseñanzas medias de la ciudad de Granada (España), para establecer una muestra representativa (error al 0,02; I.C=95,5\%), se emplearon técnicas de estratificación y proporcionalidad, considerándose tipo de rama académica (ciclos formativos y bachillerato) y sexo (hombres-mujeres). Un total de 1.164 estudiantes participaron en este trabajo, con un rango de edad de 17 a 30 años $(M=18,24$ años; $D T=1,258)$, y se obtuvo un error muestral del 0,02 en el total de la muestra y por estratos del 0,03 para bachillerato y 0,04 para ciclos formativos. El estudio cumplió con las normas éticas del Comité de Investigación y Declaración de Helsinki de 1975. Se contó en todos los casos con el consentimiento informado de participantes y se respetó el resguardo a la confidencialidad.

\section{Variables e instrumentos}

Esta investigación tomó como referencia las siguientes variables e instrumentos de medida:

- Rama de estudio, categorizado en bachillerato y ciclos formativos.

- Género, según fuese masculino o femenino.

- Nota media, estructurado en tres niveles: aprobado (5 a 6,9), notable $(7$ a 8,9$)$ y sobresaliente (9 a 10).

- Titulación de elección con limitación o sin esta, para obtener la información en la población sobre las preferencias profesionales se utilizó el cuestionario de Intereses académicos profesionales (CIBAP) de Hernández (2004), concretamente lo referente a áreas vocacionales: estudios y profesiones. Este instrumento consta de 19 cuestiones referidas a la descripción de estudios universitarios, ciclos formativos y profesiones, solicita valorar el grado de interés, determinado a través de una escala Likert de cinco opciones ( $\mathrm{A}=$ Rechazo; $\mathrm{B}=$ No me gusta; $\mathrm{C}=$ Me es indiferente o tengo dudas; $\mathrm{D}=$ Me gusta bastante y $\mathrm{E}=$ Es de mis preferidas). En función de lo planteado por Martínez-Martínez (2013) se desarrolla el ámbito de conocimiento de la titulación universitaria a elegir en el próximo curso tanto con la posibilidad de elección sin limitación (económica, familiar o económica) como con limitación, para ello y siguiendo las opciones de titulaciones universitarias se plantearon las siguientes especialidades (arqueología, arquitectura, bellas artes, biología, ciencias ambientales, ciencias del trabajo, ciencias políticas, comunicación audiovisual, criminología, derecho, económicas, empresariales, enfermería, farmacia, filología, 
doi: http://dx.doi.org/10.15359/ree.20-1.14

URL: http://www.una.ac.cr/educare

CORREO: educare@una.cr

filosofía, física-química, fisioterapia, inef, informática, ingeniería, literatura, logopeda, magisterio, matemáticas, medicina, militar, música, odontología, óptica, pedagogía, periodismo, psicología, trabajo social, traductores, turismo, veterinaria, y otras).

\section{Procedimiento}

Para la recogida de los datos se contactó, a través de la Facultad de Ciencias de la Educación de la Universidad de Granada, la totalidad de centros educativos que impartían docencia en bachillerato o ciclos formativos. A aquellos centros que dieron su autorización se les informó del protocolo de recogida de datos y se adjuntó una carta de consentimiento para las personas responsables de estudiantes que no alcanzaban la mayoría de edad, a mayores de edad se les pidió su propio consentimiento.

Los investigadores e investigadoras se hallaron presentes durante la recogida de datos informando ante cualquier duda que pudiese surgir, se debe destacar que la toma de datos se desarrolló sin ningún tipo de problema o anormalidad a reseñar y que se eliminaron 274 encuestas por no encontrarse completas en sus respuestas.

Para el análisis de los datos se utilizó el programa estadístico software SPSS 20.0., mediante la utilización de descriptivos y tablas de contingencia para establecer las diferencias entre las distintas variables: género, rama académica, nota media o titulación con limitación o sin esta.

\section{Resultados}

Comenzando el análisis descriptivo de 1.164 participantes, respecto a las variables seleccionadas, se constata que $488(41,9 \%)$ jóvenes eran varones y $676(58,1 \%)$ eran féminas, la mayor parte se encontraban estudiando bachillerato $(58,6 \%$; $n=682)$, y el resto $482(41,4 \%)$ cursaba ciclos formativos de grado superior, asimismo en lo que respecta a los parámetros de tipo académico, más de la mitad de participantes $(51,7 \% ; n=602)$ tenía notable, seguido de un $34,5 \%$ ( $n=401$ ) aprobado y, por último, únicamente un 13,8\% (n=161) obtuvo calificación de sobresaliente.

Con respecto al alumnado y la opción de sin limitación de tipo económica, de nota o familiar para cursar una titulación universitaria, eligen estudiar más que cuando se presenta alguna limitación ( $89,2 \% ; n=1038$ frente al 83\%; n=966); asimismo para este estudio se escogen las diez especializadas más valoradas por los grupos estudiantiles, destacando la similitud entre titulaciones, excepto la fisioterapia $(2,9 \% ; n=34)$ sin limitación y biología $(2,6 \% ; n=30)$ con limitación que son las que cambian; en cuanto a la preferencia de titulación universitaria sin limitación, las preferidas son, en este orden: magisterio $(11,2 \% ; n=130)$, medicina $(9,2 \% ; n=107)$ e ingeniería $(8,8 \%$; $\mathrm{n}=103$ ), en el caso de tener algún condicionante de tipo económico, nota de corte o familiar (con limitación), las tres especialidades universitarias con mayor demanda son las siguientes: magisterio $(12,5 \% ; n=145)$, ingeniería $(7,6 \% ; n=88)$ y enfermería $(6,4 \% ; n=75)$ (ver tabla 1$)$.

6

Asunción Martínez-Martínez, Félix Zurita-Ortega, Manuel Castro-Sánchez, Ramón Chacón-Cuberos, María Angustias Hinojo-Lucena y Tamara Espejo-Garcés (c) (1) (3) 
doi: http://dx.doi.org/10.15359/ree.20-1.14

URL: http://www.una.ac.cr/educare

CORREO: educare@una.cr

Tabla 1

Descriptivos de las variables

\begin{tabular}{|c|c|c|c|}
\hline \multirow{2}{*}{ Género } & Masculino & \multicolumn{2}{|l|}{$41,9 \%(n=488)$} \\
\hline & Femenino & \multicolumn{2}{|l|}{$58,1 \%(\mathrm{n}=676)$} \\
\hline \multirow{2}{*}{ Rama de estudio } & Bachillerato & \multicolumn{2}{|l|}{$58,6 \%(\mathrm{n}=682)$} \\
\hline & Ciclos formativos & \multicolumn{2}{|l|}{$41,4 \%(n=482)$} \\
\hline \multirow{3}{*}{ Nota media } & Aprobado & \multicolumn{2}{|l|}{$34,5 \%(n=401)$} \\
\hline & Notable & \multicolumn{2}{|l|}{$51,7 \%(\mathrm{n}=602)$} \\
\hline & Sobresaliente & \multicolumn{2}{|l|}{$13,8 \%(\mathrm{n}=161)$} \\
\hline \multirow{12}{*}{ Titulación universitaria } & & Sin Limitación & Con Limitación \\
\hline & No estudiar & $10,8 \%(n=126)$ & $17 \%(n=198)$ \\
\hline & Medicina & $9,2 \%(n=107)$ & $5,0 \%(\mathrm{n}=58)$ \\
\hline & Magisterio & $11,2 \%(n=130)$ & $12,5 \%(n=145)$ \\
\hline & Derecho & $2,7 \%(n=31)$ & $3,2 \%(n=37)$ \\
\hline & Enfermería & $6,3 \%(n=73)$ & $6,4 \%(n=75)$ \\
\hline & Fisioterapia-biología & $2,9 \%(n=34)$ & $2,6 \%(n=30)$ \\
\hline & Económicas & $4,0 \%(n=47)$ & $4,5 \%(\mathrm{n}=52)$ \\
\hline & Informática & $3,3 \% \quad(n=38)$ & $3,6 \%(n=42)$ \\
\hline & INEF & $4,9 \%(n=57)$ & $4,9 \%(n=57)$ \\
\hline & Psicología & $4,7 \%(\mathrm{n}=55)$ & $4,6 \%(n=53)$ \\
\hline & Ingeniería & $8,8 \%(n=103)$ & $7,6 \%(n=88)$ \\
\hline
\end{tabular}

Al establecer las relaciones entre las variables de tipo género, con la rama de estudios, calificación media y elección de especialidad universitaria (tabla 2), en todos los casos los análisis estadísticos fijaron diferencias estadísticamente significativas $\left(p \leq .005^{* *}\right)$, ellos son mayoría en los ciclos formativos (44,9\% frente al 38,9\%), mientras las participantes femeninas están más matriculadas en bachillerato (61,1\% frente al $55,1 \%)$.

En lo que se refiere a las calificaciones medias debemos puntualizar cómo los participantes masculinos obtienen más aprobados que las estudiantes femeninas (39,1\% frente al $31,1 \%)$, esta tendencia cambia en la calificación de notable ( $54,6 \%$ en chicas y $47,7 \%$ para chicos), y en la nota de sobresaliente los resultados son similares. 
doi: http://dx.doi.org/10.15359/ree.20-1.14

URL: http://www.una.ac.cr/educare

CORREO: educare@una.cr

En las participantes femeninas existe una predominancia hacia ciertas titulaciones sin limitación más que en los hombres, de esta forma en estudios de medicina, magisterio, enfermería o psicología los porcentajes son mayores, situación que se invierte en carreras como las de informática, INEF o ingeniería, donde los varones puntúan mucho más alto que las alumnas; en el resto de carreras (económicas, derecho y fisioterapia) los valores son idénticos, en lo que refiere a la elección de una titulación con limitaciones económicas, de nota o familiar, cambiando únicamente el grado de biología, más demandado por ellas.

Tabla 2

Género y rama según elección del itinerario curricular de último curso

\begin{tabular}{|c|c|c|c|}
\hline \multirow[b]{2}{*}{ Rama } & \multicolumn{2}{|c|}{ Género } & \multirow[b]{2}{*}{$X^{2}$} \\
\hline & Masculino & Femenino & \\
\hline Ciclos formativos & $44,9 \%(n=219)$ & $38,9 \%(n=263)$ & $\mathrm{p}=.041^{* *}$ \\
\hline Bachillerato & $55,1 \%(n=269)$ & $61,1 \%(n=413)$ & \\
\hline Nota media & & & $x^{2}$ \\
\hline Aprobado & $39,1 \%(n=191)$ & $31,1 \%(n=210)$ & $\mathrm{p}=.016^{* *}$ \\
\hline Notable & $47,7 \%(n=233)$ & $54,6 \%(n=369)$ & \\
\hline Sobresaliente & $13,1 \%(n=64)$ & $14,3 \%(n=97)$ & \\
\hline Titulación sin limitación o con ella & Sin/Con & Sin/Con & $x^{2}$ \\
\hline Medicina & $26,2 \%(n=28) / 25,9 \%(n=15)$ & $73,8 \%(n=79) / 74,1 \%(n=43)$ & $\mathrm{p}=.000^{* *}$ \\
\hline Magisterio & $16,9 \%(n=22) / 17,9 \%(n=26)$ & $83,1 \%(n=108) / 82,1 \%(n=119)$ & \\
\hline Derecho & $54,8 \%(n=17) / 43,2 \%(n=16)$ & $45,2 \%(n=14) / 56,8 \%(n=21)$ & \\
\hline Enfermería & $21,9 \%(n=16) / 21,3 \%(n=16)$ & $78,1 \%(n=57) / 78,7 \%(n=59)$ & \\
\hline Fisioterapia-biología & $27,8 \%(n=5) / 13,3 \%(n=4)$ & $72,2 \%(n=13) / 86,7 \%(n=26)$ & \\
\hline Económicas & $44,7 \%(n=21) / 42,3 \%(n=22)$ & $55,3 \%(n=26) / 57,7 \%(n=30)$ & \\
\hline Informática & $92,1 \%(\mathrm{n}=35) / 90,5 \%(\mathrm{n}=38)$ & $7,9 \%(n=3) / 9,5 \%(n=4)$ & \\
\hline INEF & $82,5 \%(n=47) / 84,2 \%(n=48)$ & $17,5 \%(n=10) / 15,8 \%(n=9)$ & \\
\hline Psicología & $12,7 \%(n=7) / 13,2 \%(n=7)$ & $87,3 \%(n=48) / 86,8 \%(n=46)$ & \\
\hline Ingeniería & $85,4 \%(n=88) / 84,1 \%(n=74)$ & $14,6 \%(n=15) / 15,9 \%(n=14)$ & \\
\hline
\end{tabular}

En cuanto a la rama y su relación con las notas académicas y las elecciones de titulaciones con limitación o sin esta, se establecieron diferencias estadísticamente significativas ( $\left.p \leq .005^{* *}\right)$ en todos los casos como se aprecia en la tabla 3. De esta forma, en la nota de aprobado, los 
doi: http://dx.doi.org/10.15359/ree.20-1.14

URL: http://www.una.ac.cr/educare

CORREO: educare@una.cr

grupos participantes de bachillerato $(41,2 \%)$ sacan más alto porcentaje que los de ciclos formativos $(24,9 \%)$, mientras que en notable y sobresaliente, los de ciclos formativos tienen mayor porcentaje que los de bachillerato. Asimismo, en los procedentes de ciclos formativos existe una predominancia hacia ciertas titulaciones sin limitación más que los de bachillerato, de esta forma en estudios de enfermería e ingeniería se produce este hecho, asimismo en alumnos y alumnas que están en bachillerato los porcentajes son mayores, en carreras como derecho o INEF; en el resto de carreras (económicas, medicina, magisterio, psicología, informática y fisioterapia) los valores son idénticos; esta tendencia cambia ligeramente en las con limitación, donde el alumnado de ciclos formativos puntúan de manera más alta la titulación de enfermería, por el contrario el de segundo de bachillerato valora más las titulaciones de medicina, derecho e INEF. En el resto de titulaciones universitarias los datos se mantuvieron uniformes.

Tabla 3

Rama de estudios según calificación y elección de titulación

\begin{tabular}{|c|c|c|c|}
\hline & \multicolumn{2}{|c|}{ Rama de estudios } & \multirow[b]{2}{*}{$X^{2}$} \\
\hline & Ciclos formativos & Bachillerato & \\
\hline \multicolumn{4}{|l|}{ Nota media } \\
\hline Aprobado & $24,9 \%(n=120)$ & $41,2 \%(n=281)$ & \\
\hline Notable & $58,9 \%(n=284)$ & $46,6 \%(n=318)$ & $\mathrm{p}=.000^{* *}$ \\
\hline Sobresaliente & $16,2 \%(n=78)$ & $12,2 \%(n=83)$ & \\
\hline Titulación con limitación o sin esta & Sin/Con & Sin/Con & $X^{2}$ \\
\hline Medicina & $36,4 \%(n=39) / 22,4 \%(n=13)$ & $63,6 \%(n=68) / 77,6 \%(n=45)$ & $\mathrm{p}=.000^{* *}$ \\
\hline Magisterio & $40,8 \%(n=53) / 37,9 \%(n=55)$ & $59,2 \%(n=77) / 62,1 \%(n=90)$ & \\
\hline Derecho & $12,9 \%(n=4) / 18,9 \%(n=7)$ & $87,1 \%(n=27) / 81,1 \%(n=30)$ & \\
\hline Enfermería & $69,9 \%(n=51) / 64 \%(n=48)$ & $30,1 \%(n=22) / 36 \%(n=27)$ & \\
\hline Fisioterapia-biología & $16,7 \%(n=3) / 26,7 \%(n=8)$ & $83,3 \%(n=15) / 73,3 \%(n=22)$ & \\
\hline Económicas & $36,2 \%(n=17) / 34,6 \%(n=18)$ & $63,8 \%(n=30) / 65,4 \%(n=34)$ & \\
\hline Informática & $57,9 \%(n=22) / 57,1 \%(n=24)$ & $42,1 \%(n=16) / 42,9 \%(n=18)$ & \\
\hline INEF & $14 \%(n=8) / 15,8 \%(n=9)$ & $80,7 \%(n=49) / 84,2 \%(n=48)$ & \\
\hline Psicología & $29,1 \%(n=16) / 22,6 \%(n=12)$ & $70,9 \%(n=39) / 77,4 \%(n=41)$ & \\
\hline Ingeniería & $56,3 \%(n=58) / 52,3 \%(n=46)$ & $43,7 \%(n=45) / 47,7 \%(n=42)$ & \\
\hline
\end{tabular}


doi: http://dx.doi.org/10.15359/ree.20-1.14

URL: http://www.una.ac.cr/educare

CORREO: educare@una.cr

Para finalizar se detectó igualmente falta de correspondencia entre las calificaciones y la elección de titulación ( $\mathrm{p} \leq .005^{* *}$ ) como se desprende de las cifras en la tabla 4 , de esta manera entre los grupos estudiantiles que pretendían cursar el próximo año una titulación sin ningún tipo de condicionante, los que obtienen aprobado se decantaron mayoritariamente por las titulaciones de magisterio $(44,6 \%)$, derecho $(58,1 \%), \operatorname{INEF}(54,4 \%)$ y psicología $(52,7 \%)$, asimismo los que tenían calificaciones de notable, preferían los grados de medicina (50,5\%), magisterio (45,4\%), enfermería (67,1\%), fisioterapia $(44,4 \%)$, económicas $(53,2 \%)$, informática $(68,4 \%)$ e ingeniería $(49,5 \%)$ y entre los que tenían sobresaliente, preferían estudiar medicina $(35,5 \%)$ y fisioterapia $(27,8 \%)$.

En cuanto a las calificaciones de participantes y la elección de titulación que pretendían cursar el próximo año con algún tipo de limitación (nota media, económicas, etc.), se generó gran disparidad de cifras. De esta forma, estudiantes con aprobado querían estudiar las titulaciones de derecho $(54,1 \%)$ e INEF $(54,4 \%)$, puntualizar que los grados de magisterio $(46,2 \%$ y $46,9 \%)$ y psicología $(47,2 \%$ y 49,1\%) plantearon datos similares entre aprobado y notable, entre estos últimos también tenemos los grados de enfermería (66,7\%), biología (63,3\%), económicas (55,8\%), informática (69\%) e ingeniería (51,1\%). Entre los quienes tenían sobresaliente, se decantaban claramente por estudiar medicina (50\%).

Tabla 4

Calificación y elección de titulación

\begin{tabular}{lccc}
\hline & & Calificaciones & \\
\hline & Aprobado & Notable & Sobresaliente \\
\hline Titulación & Sin/Con & Sin/Con & Sin/Con \\
\hline Medicina & $14 \%(n=15) / 5,2 \%(n=3)$ & $50,5 \%(n=54) / 44,8 \%(n=26)$ & $35,5 \%(n=38) / 50 \%(n=29)$ \\
Magisterio & $44,6 \%(n=58) / 46,2 \%(n=67)$ & $45,4 \%(n=59) / 46,9 \%(n=68)$ & $10 \%(n=13) / 6,9 \%(n=10)$ \\
Derecho & $58,1 \%(n=18) / 54,1 \%(n=20)$ & $41,9 \%(n=13) / 40,5 \%(n=15)$ & $0 \%(n=0) / 5,4 \%(n=2)$ \\
Enfermería & $21,9 \%(n=16) / 26,7 \%(n=20)$ & $67,1 \%(n=49) / 66,7 \%(n=50)$ & $11 \%(n=8) / 6,7 \%(n=5)$ \\
Fisioterapia-Biología & $27,8 \%(n=5) / 10 \%(n=3)$ & $44,4 \%(n=8) / 63,3 \%(n=19)$ & $27,8 \%(n=5) / 26,7 \%(n=8)$ \\
Económicas & $36,2 \%(n=17) / 34,6 \%(n=18)$ & $53,2 \%(n=25) / 55,8 \%(n=29)$ & $10,6 \%(n=5) / 9,6 \%(n=5)$ \\
Informática & $23,7 \%(n=9) / 23,8 \%(n=10)$ & $68,4 \%(n=26) / 69 \%(n=29)$ & $7,9 \%(n=3) / 7,1 \%(n=3)$ \\
INEF & $54,4 \%(n=31) / 54,4 \%(n=31)$ & $40,4 \%(n=23) / 40,4 \%(n=23)$ & $5,3 \%(n=3) / 5,3 \%(n=3)$ \\
Psicología & $52,7 \%(n=29) / 47,2 \%(n=25)$ & $43,6 \%(n=24) / 49,1 \%(n=26)$ & $3,6 \%(n=2) / 3,8 \%(n=2)$ \\
Ingeniería & $37,9 \%(n=39) / 34,1 \%(n=30)$ & $49,5 \%(n=51) / 51,1 \%(n=45)$ & $12,6 \%(n=13) / 14,8 \%(n=13)$ \\
\hline
\end{tabular}




\section{Discusión}

Las 1.164 personas participantes de último curso de bachillerato y ciclos formativos de grado superior analizadas en este trabajo, con proporciones homogéneas por género, tienen las mismas características encontradas en otros estudios realizados en la geografía española (Cepero, 2009; Naranjo, 1993; Rodríguez-Moreno, Delgado, Estruch, Quero y Riart 1986; Sanjuán, 2010; Toscano, 2004) e incluso se señala la importancia de publicaciones de esta índole (Capilla, 2009; Castilla, 2003) y que estos se realicen desde las perspectiva de la institución educativa. Una gran parte de la muestra recogida provenía de bachillerato mientras que de los ciclos formativos se encontró un alto absentismo de contestación hacía las preguntas realizadas, esto concuerda con lo reportado por Toscano (2004) que matiza que entre estudiantes de bachillerato todo lo relacionado con conocer el futuro de demandas universitarias, tutoriales y de organización les crea numerosas atenciones; sin embargo, en el contexto de los ciclos formativos estas expectativas no son tan altas, por lo que en ocasiones deciden no acudir a encuestas de esta índole.

La mayoría de participantes de esta investigación obtuvo calificaciones medias de notable, si bien estas cifras están en desacuerdo con lo reportado en el último Informe 2012 (OCDE, 2013) que destacaba que España se encuentra en los últimos lugares del contexto mundial de los países desarrollados en rendimiento académico, y los datos señalaron que la opción de continuar estudios universitarios superiores sin limitación de tipo económica, de nota o familiar eran mayoritarios a cuando existía algún tipo de limitación.

Entre las titulaciones con mayor demanda se indican los grados de magisterio, medicina e ingeniería, siempre y cuando no existiese ningún tipo de condicionante económico, nota de corte o familiar, y en el caso de existir alguna limitación, eran las anteriormente citadas con el cambio de enfermería por medicina. Entendemos que los sujetos analizados se postulan en tres ámbitos del conocimiento como son las ciencias sociales (magisterio), técnicas (ingeniería) y ciencias de la salud (medicina y enfermería), lo que corrobora que el aspecto vocacional es esencial a la hora de elegir estudios superiores (Cepero, 2009; Martínez-Rodríguez y Carmona, 2010), asimismo esta afirmación es aún más patente en el contexto de las ciencias de la salud, pues cuando no existe limitación el alumnado pretende estudiar medicina, sin embargo, al existir problemas de calificaciones estos incluyen la titulación de enfermería, si bien el campo del saber es el mismo. Asimismo, el orientarse a estos grados universitarios o carreras parece ser porque están entre las mejor remuneradas y es el principal aspecto extrínseco (Elejabeitia, 1995; Serrano, Montañés y La Torre, 1999) y es, como puntualiza García-Sedeño (2002), una visión de la formación dirigida hacia el resultado como un medio para conseguir una meta.

Por tanto, las titulaciones de índole sanitario (Lora-López, 2008), unidas a las ingenierías (Fernández y Vaquero, 2006) suelen ser de las más solicitadas por las expectativas futuras que plantean así como porque popularmente han tenido bastante prestigio social. También 
doi: http://dx.doi.org/10.15359/ree.20-1.14

URL: http://www.una.ac.cr/educare

CORREO: educare@una.cr

debemos hacernos eco de las manifestaciones que realiza Gordaliza (2010) cuando indica que en estos últimos años las titulaciones derivadas del ámbito de las facultades de ciencias se ven mermadas en el número de alumnado a consecuencia del descrédito que tienen en la actualidad las titulaciones de ciencias (matemáticas, física, química, etc.).

Los varones son mayoría en los ciclos formativos y obtienen un mayor número de aprobados que las participantes femeninas que se encuentran sobretodo matriculadas en bachillerato y tienen mayor número de notables. Numerosas investigaciones (Alonso, 2009; Cepero, 1997; Mosteiro y Méndez, 1998; Parra y Oliva, 2002; Rivas, 1998) indican que la elección y aspiraciones académicas de adolescentes parecen estar condicionadas de forma importante por los estereotipos de género existentes en nuestra sociedad.

En las participantes femeninas existe una predominancia hacía las titulaciones de medicina, magisterio, enfermería o psicología, mientras los varones se decantan por la informática, INEF o ingeniería. Estos datos denotan que entre las mujeres priman áreas vocacionales de sanidad y enseñanza. La vocación va encaminada hacía estas opciones, e intuimos que esta situación viene determinada por dos factores: por un lado, los parámetros laborales, ya que ambas opciones desencadenan en numerosas opciones académicas y laborales; y por otra parte, la predisposición a dedicarse a la atención y al servicio de los demás como señalan Alonso (2009); Barra (2002); Sadurní y Rostan (2004).

En cuanto a las notas con las que acceden a último curso, la mitad de participantes tenía una calificación de notable y un alto porcentaje de aprobado, lo que denota que a mayor curso académico las calificaciones parecen ir mejorando. Entendemos que este hecho viene propiciado porque en estos cursos la enseñanza no es obligatoria, por lo que la mayor parte del alumnado ha escogido libremente la opción de estudiar y esto repercute de una manera favorable en el rendimiento académico. Fuente de la y Justicia (2001) y Herrera, Nieto, Rodríguez y Sánchez (1999) señalan que las alumnas presentan mejores niveles de motivación académica en general, sobre todo en las etapas anteriores al acceso a la universidad, estando más motivadas para mantener una conducta de estudio.

En el estudiantado de último curso de ciclos formativos existe una predominancia hacia las titulaciones de enfermería e ingeniería, mientras que en el alumnado de bachillerato se postulan más en carreras como derecho o INEF; en el resto de grados (económicas, medicina, magisterio, psicología, informática y fisioterapia/biología) los valores son idénticos.

Asimismo se debe señalar que tradicionalmente se ha observado la existencia de ciertas titulaciones donde las notas de corte eran bastante altas como medicina o fisioterapia, los motivos e intenciones de elección de la titulación son los que señalan Martínez, Gimeno y Rocabert (1998) en Cepero (2009); Fernández-Poncela (2000) o León y Gambara (2005) que manifiestan tener un buen sueldo para mantener a la familia y la economía del hogar o tener 
buenos salarios, esto concuerda con lo que sucede en estudiantes de nuestro estudio donde los que tenían notas medias más altas querían estudiar medicina, mientras que las titulaciones de magisterio, derecho e INEF eran su mayoría demandadas por estudiantes con aprobado de nota media. A este respecto en enseñanzas de ciencias sociales y según la tipología de estudiantes que piensa acceder a estos grados las calificaciones son inferiores como citan Langa (2003) y Prieto-Zurita (2010), del mismo modo las de mejor puntuación vienen condicionadas a ser exclusivas del bloque de salud (medicina, fisioterapia, enfermería,...). En referencia a esto, Castro (1998) expone que los grupos adolescentes eligen una profesión o titulación para su realización personal, y no solo como medio de subsistencia, por lo que tanto, en las opciones biomédicas como técnicas existe un aumento de personas con tradición de estudios superiores universitarios.

Los datos y valores arrojados en este estudio son parecidos entre la elección con limitación o sin esta, por lo que se pone de manifiesto que en los estudiantes prima la vocación frente al interés profesional (Martínez-Martínez, 2013).

Para finalizar, debemos señalar que como en todo estudio se presentan una serie de limitaciones, en este han venido producidas porque una parte considerable de estudiantes de ciclos formativos rehusaron la realización de este estudio, del mismo modo también podríamos haber incluido cuestiones hacia los padres y madres para constatar si la información recogida era la veraz y tener en cuenta algunos supuestos como abandono en los últimos cursos y motivos de este.

\section{Conclusiones}

Como principales conclusiones de este estudio extraemos que las titulaciones de medicina, magisterio e ingenierías son las más demandadas sin limitación mientras que con limitación cambia medicina por enfermería; asimismo el alumnado de último curso de Granada tenía una nota media de notable, los varones se decantan más por los ciclos formativos, presentando peores calificaciones, y las mujeres prefieren el bachillerato, con notas medias ligeramente superiores como ha quedado reflejado en el estudio, del mismo modo queda constatada la influencia que tienen las calificaciones académicas, la rama de estudios y el género en la elección de titulación con limitación o sin tal, así ellas se postulan por carreras de carácter más altruista en grados de ciencias de la salud y sociales y con notas más altas mientras que los estudiantes masculinos prefieren titulaciones más técnicas y con peores calificaciones.

Este tipo de estudio pone de manifiesto la necesidad de involucrar la figura del orientador u orientadora como aspecto esencial en el estudiantado para su devenir formativo y laboral futuro. 
doi: http://dx.doi.org/10.15359/ree.20-1.14

URL: http://www.una.ac.cr/educare

CORREO: educare@una.cr

\section{Referencias}

Alonso, A. (Abril, 2009). Orientación no sexista. VI Jornadas de Coeducación "Rompiendo estereotipos". Granada: Centro de Educación del Profesorado de Granada (CEP).

Barra, E. (2002). Influencia del sexo y de la tipificación de rol sexual sobre las actitudes hacia la homosexualidad masculina y femenina. Revista Latinoamericana de Psicología, 34(3), 275284. Recuperado de http://www.redalyc.org/pdf/805/80534307.pdf

Bisquerra, R. (1992). Orientación psicopedagógica para la prevención y el desarrollo. Barcelona: Boixareu.

Blanco, A., Mercado, C. y Prado, A. (Diciembre, 2012). Perfil y motivación de la juventud emprendedora española. Revista de Estudios de Juventud, 99, 22-34. Recuperado de http:// www.injuve.es/sites/default/files/2013/18/publicaciones/Cap\%202\%20\%20INJUVE\%20 n\%C2\%BA\%2099-2.pdf

Capilla, R. (2009). Análisis estratégico de los estudios TIC en la Universidad Politécnica de Valencia (Tesis doctoral). Universidad Politécnica de Valencia. https://riunet.upv.es/bitstream/ handle/10251/5767/tesisUPV3043.pdf?sequence=1\&isAllowed=y

Castilla, M. T. (2003). Perfil profesional e itinerarios de formación e inserción profesional del psicopedagogo (Tesis doctoral). Universidad de Granada.

Castro, M. (1998). O perfil socioloxico do estudiante da Diplomatura de Educación Social da Universidade de Santiago de Compostela [El perfil sociológico de los estudiantes de la diplomatura de Educación ocial de la Universidad de Santiago de Compostela] (Tesis de licenciatura). Universidad de Santiago de Compostela.

Cepero, A. B. (1997). Intervención psicopedagógica diferenciada por género en el proceso de orientación vocacional/profesional. Revista Galego/Portuguesa de Psicología eEducación, 1(1), 377-386. Recuperado de http://hdl.handle.net/2183/6604

Cepero, A. B. (2009). Las preferencias profesionales y vocacionales del alumnado de secundaria y formación profesional específica (Tesis doctoral). Universidad de Granada. Recuperado de http://hera.ugr.es/tesisugr/18751362.pdf

Cordón, A, Cordón, E y García, F. (2012). Evidencias empíricas en el proceso de elección de los estudios universitarios. Enseñanza \& Teaching: Revista interuniversitaria de didáctica, 30(2), 45-62. Recuperado de http://campus.usal.es/ revistas trabajo/index.php/0212-5374/ article/view/9310/9606

Cortés, P. A. y Conchado, A. (2012). Los contextos parentales y académicos y los valores laborales en la toma de decisiones en bachillerato. ESE. Estudios sobre Educación, 22, 93-114. Recuperado de http://dadun.unav.edu/bitstream/10171/22632/2/ese-22\%2093-114.pdf 
Domínguez, G., Álvarez, F. J. y López, A. M. (Mayo-agosto, 2013). Acción tutorial y orientación en el periodo de transición de la educación secundaria a la universidad. La orientación al alumno de nuevo ingreso. REDU, Revista de Docencia Universitaria, 11(2), 221-241.

Elejabeitia, C. (1995). Las pulsiones sociales de la variable sexo en la elección de las carreras: Pioneras y marginados. Madrid: Ministerio de Educación.

Fernández-Poncela, A. (2000). Elecciones, cámaras y mujeres. Barcelona: Servicio de publicaciones de la Universidad Autónoma de Barcelona.

Fernández, S. y Vaquero, A. (Marzo, 2006). El grado de adecuación con la oferta a la demanda de titulaciones en el sistema universitario de Galicia. Revista Galega do Ensino, 14(48), 451 470. Recuperado de http://dialnet.unirioja.es/ejemplar/131650

Fuente de la, J. y Justicia, F. (2001). Diferencias de género en las técnicas de aprendizaje utilizadas por los alumnos universitarios. Revista Gallego-Portuguesa de Psicoloxía e Educación, 7(5), 38-45. Recuperado de http://ruc.udc.es/bitstream/2183/6721/1/RGP 5.20.pdf

Gallo, C., González, C. y Salinero, J. J. (2010). Estudio comparativo de las motivaciones, percepciones y expectativas de futuro entre estudiantes de C.C.A.F.D., de la Universidad Camilo José Cela (España) y la Universidad de Hertfordshire (Inglaterra). Journal of Sport and Health Research, 2(3), 253-260. Recuperado de http://www.journalshr.com/papers/Vol\%202 N\%203/ V02 3 06.pdf

García-Sedeño, M. (2002). Modelo de cinco factores. Revista de la federación Española de Asociaciones de Psicología, 43, 134-136.

Gordaliza, A. (Febrero, 2010). Adapting the spanish degree on statistics to the European space for higher education [Adaptación de los grados españoles a las estadísticas para el espacio europeo de educación superior]. Boletín de Estadística e Investigación Operativa, 26(1), 97102. Recuperado de http://www.seio.es/BEIO/files/BEIOVol26Num1OP3-Web.pdf

Hernández, V. (2004). Evaluación de los intereses básicos académico profesionales de los estudiantes de secundaria. REOP, 15(1) 117-141. Recuperado de http://www.uned.es/ reop/pdfs/2004/15-1-1\%20-\%20Vicente\%20Hernandez\%20Franco.PDF

Herrera, M. E., Nieto, S., Rodríguez, M. J. y Sánchez, M. C. (1999). Factores implicados en el rendimiento académico de los alumnos Universidad de Salamanca. Revista de Investigación Educativa, 17(2), 413-421. Recuperado de http://revistas.um.es/rie/article/ view/121981/114661

Langa, D. (2003). Los estudiantes y sus razones prácticas: Heterogeneidad de estrategias de estudiantes universitarios según clase social (Tesis doctoral). Universidad Complutense de Madrid. Recuperado de http://eprints.ucm.es/4709/1/T26504.pdf 
doi: http://dx.doi.org/10.15359/ree.20-1.14

URL: http://www.una.ac.cr/educare

CORREO: educare@una.cr

León, O. G. y Gambara, H. (2005). Decision aiding tool for university subjects. D.A.T.U.S. Herramienta para la elección de asignaturas optativas universitarias. Psicothema, 17(1), 164-168. Recuperado de http://www.redalyc.org/articulo.oa?id=72717126

Llosa, S., Acin, A., Cragnolino, E., y Lorenzatti, M. (2012). La demanda potencial y la demanda efectiva en educación de jóvenes y adultos: Primera aproximación al enfoque cuantitativo y cualitativo de la biografía educativa. Reflexiones en torno al significado atribuido a la educación y la capacitación laboral. Cuadernos de Educación, 2(2), 175-187. Recuperado de http://www.revistas.unc.edu.ar/index.php/Cuadernos/article/view/661/625

Lora-López, P. (Abril-junio, 2008). Reflexiones sobre el grado y postgrado de enfermería: La investigación en enfermería. Index de Enfermería, 17(2), 85-86. Recuperado de http://www. index-f.com/index-enfermeria/v17n2/6648.php

Lorente, R., Calvo, R., Redondo, A., Gallego, J. R., Benedito, M. A. y Cano, F. J. (2011). La importancia del conocimiento de las salidas profesionales en la elección y al cursar una titulación. Proyecto mercado laboral de los egresados en relaciones laborales. Documento presentado en la Conferencia de Universitat de Girona. Institut de Ciències de l'Educació Josep Pallach. Recuperado de http://hdl.handle.net/10256/3667

Lorenzo-Moledo, M., Argos, J., Hernández, J. y Vera, J. (2014). El acceso y la entrada del estudiante a la universidad: Situación y propuestas de mejora facilitadoras del tránsito. Educación XX1, 17(1), 15-38. Recuperado de http://www.redalyc.org/articulo.oa?id=70629509001

Mantero, M. (2010). Orientación educativa y culturas docentes en la educación postobligatoria: Estudio de las relaciones entreel orientadory el profesorado en niveles de formación profesional específica (Tesis doctoral). España, Universidad de Huelva. Recuperado de http://redined. mecd.gob.es/xmlui/handle/11162/2936

Martínez-Martínez, A. (2013). La orientación como actividad educativa y vocacional en los itinerarios curriculares del alumnado de bachiller y formación profesional y su inclusión en el mercado laboral (Tesis doctoral). España, Universidad de Granada. Recuperado de http:// digibug.ugr.es/handle/10481/30837\#.VhVIYalspdY

Martínez-Martínez, A. y Zurita, F. (2014). El rol que tiene la influencia familiar y su nivel académico en los itinerarios curriculares del estudiante de último curso. Educatio Siglo XXI, 32(2), 267286. doi: http://dx.doi.org/10.6018/j/202251

Martínez-Rodríguez, F. M. y Carmona, G. (Junio, 2010). Test de factores sociopersonales para la inserción laboral de los jóvenes: Un instrumento para la evaluación y la formación. ESE. Estudios sobre Educación, 18, 115-138. doi: http://dspace.unav.es/dspace/ bitstream/10171/9823/2/ESE 18 6.pdf 
Martín-Moreno, M. J. (1996). Sistema de experto de orientación vocacional profesional (Un procedimiento informatizado de ayuda) (Tesis doctoral). España, Universidad de Madrid. Recuperado de http://biblioteca.ucm.es/tesis/19972000/S/4/S4016401.pdf

Mosteiro, M. J. y Méndez, M. J. (1998). La elección de opciones académicas en el bachillerato y la formación profesional desde una perspectiva de género. Revista de Estudios e Investigación en Psicología e Educación, 2(3), 16-24.

Naranjo, M. A. (1993). Estilo cognitivo, intereses y elección académico profesional en la orientación de los alumnos de COU (Tesis doctoral). España, Universidad Complutense de Madrid. Recuperado de http://biblioteca.ucm.es/tesis/19911996/S/5/S5000901.pdf

OCDE (2013). PISA 2012. Programa para la evaluación internacional de los alumnos. Informe español (Volumen I: Resultados y contexto). Madrid: Ministerio de Educación, Cultura y Deporte. Gobierno de España. Recuperado de http://www.mecd.gob.es/dctm/inee/ internacional/pisa2012/pisa2012lineavolumeni.pdf?documentld=0901e72b81786310

Parra, Á. y Oliva, A. (Diciembre, 2002). Comunicación y conflicto familiar durante la adolescencia. Anales de Psicología, 18(2), 215-231.

Pérez, E., Cupani, M.y Beltramino, C. (Setiembre, 2004). Adaptación del inventario de personalidad 16PF-IPIP a un contexto de orientación. Estudio Preliminar. Evaluar, 4, 23-49. Recuperado de http://www.researchgate.net/publication/237795811 Adaptacin del Inventario de Personalidad 16PF-IPIP a un Contexto de Orientacin. Estudio Preliminar

Prieto-Zurita, M. (2010). Estudio de las titulaciones de magisterio de educación física y educación infantil en el centro de enseñanza superior en humanidades y ciencias de la educación "Don Bosco" (Tesis doctoral). Universidad Complutense de Madrid. Recuperado de http:// eprints.ucm.es/10537/1/T31410.pdf

Real Decreto 676 (7 de mayo de 1993). BOE número 122, de 22 de mayo de 1993, pp. 15610 a 15614. Recuperado de http://www.boe.es/diario boe/txt.php?id=BOE-A-1993-13322

Real Decreto 1892 (14 de noviembre de 2008). BOE número 283, de 24 de noviembre de 2008, pp. 46932 a 46946. Recuperado de https://www.boe.es/diario boe/txt. php?id=BOE-A-2008-18947

Rivas, F. (1998). Psicología vocacional: Enfoques del asesoramiento. Madrid: Morata.

Rodríguez-Moreno, M. L., Delgado, L. Estruch, I., Quero, J. M. y Riart, J. (1986). Orientación profesional y acción tutorial en las enseñanzas medias. Madrid: Narcea.

Ruesga, S. M., Martín, J. L. y Pérez, L. (2010). Informe de coyuntura sobre el mercado de trabajo en España y Andalucía (Primer trimestre de 2010). Temas Laborales, 105, 131-159. Recuperado de http://dialnet.unirioja.es/ejemplar/256922 
doi: http://dx.doi.org/10.15359/ree.20-1.14

URL: http://www.una.ac.cr/educare

CORREO: educare@una.cr

Sadurní, M. y Rostan, C. (2004). La importancia de las emociones en los periodos sensibles del desarrollo. Infancia y aprendizaje, 27(1), 105-114. doi: http://dx.doi. org/10.1174/021037004772902132

Sanjuán, M. (2010). Los contenidos y las competencias del módulo de formación y orientación laboral (FOL): Visión del profesorado en Galicia. Innovación Educativa, 20, 89-103. Recuperado de http://dspace.usc.es/bitstream/10347/5000/1/07.Sanjuan.pdf

Santana, L. E., Feliciano, L. A. y Jiménez, A. B. (Setiembre-diciembre, 2012). Toma de decisiones y género en el bachillerato. Revista de Educación, 359, 357-387.

Serrano, J. P., Montañés, J. y La Torre, J. M. (Septiembre, 1999). Un modelo de intervención para la toma de decisión vocacional. La zona de división vocacional. El género como elemento de sesgo. III Congreso Internacional de Psicología y Educación: Orientación e Intervención Psicopedagógica. Santiago de Compostela.

Toscano, M. (2004). Estudio sobre la orientación académica para el tránsito del bachillerato a la Universidad de Huelva: Diseño, aplicación y evaluación de un programa de orientación para el acceso a la universidad (Tesis doctoral) Universidad de Huelva. Recuperado de http:// rabida.uhu.es/dspace/handle/10272/2784?locale-attribute $=$ fr

Villanueva, L. y Sorribes, S. (1995). Orientación vocacional y género. En tutoría y orientación. Actas, comunicaciones y talleres. V Jornadas LOGSE (pp. 391-398). Barcelona: CEDECS.

Viñuela-Jiménez, A., Rubiera-Morollón, F. y Cueto, B. (2010). An analysis of urban size and territorial location effects on employment probabilities: The spanish case [Análisis de tamaño urbano y los efectos de localización territorial sobre las posibilidades de empleo: El caso español]. Growth and Change. A Journal of Urban and Regional Policy, 41(4), 495519. doi: http://dx.doi.org/10.1111/j.1468-2257.2010.00536.x

\section{Cómo citar este artículo en APA:}

Martínez-Martínez, A., Zurita-Ortega, F., Castro-Sánchez, M., Chacón-Cuberos, R., Hinojo-Lucena, M. A. y Espejo-Garcés, T. (Enero-abril, 2016). La elección de estudio superiores universitarios en estudiantes de último curso de bachillerato y ciclos formativos. Revista Electrónica Educare, 20(1), 1-18. doi: http://dx.doi. org/10.15359/ree.20-1.14

Nota: Para citar este artículo en otros sistemas puede consultar el hipervínculo "Como citar el artículo" en la barra derecha de nuestro sitio web: http://www.revistas.una.ac.cr/index.php/EDUCARE/index 